\title{
Multiplicative Watermarking Method with the Visual Saliency Model Using Contourlet Transform
}

\author{
Jinhua Liu $\mathbb{D}^{1}{ }^{1}$ Jiawen Huang, ${ }^{1}$ and Yuanyuan Huang ${ }^{2}$ \\ ${ }^{1}$ School of Mathematical and Computer Sciences, Shangrao Normal University, Shangrao 334001, China \\ ${ }^{2}$ Department of Network Engineering, Chengdu University of Information Technology, Chengdu 610225, China
}

Correspondence should be addressed to Jinhua Liu; liujinhua_uestc@126.com

Received 17 June 2021; Accepted 27 September 2021; Published 7 October 2021

Academic Editor: Beijing Chen

Copyright (c) 2021 Jinhua Liu et al. This is an open access article distributed under the Creative Commons Attribution License, which permits unrestricted use, distribution, and reproduction in any medium, provided the original work is properly cited.

We have proposed an image adaptive watermarking method by using contourlet transform. Firstly, we have selected high-energy image blocks as the watermark embedding space through segmenting the original image into nonoverlapping blocks and designed a watermark embedded strength factor by taking advantage of the human visual saliency model. To achieve dynamic adjustability of the multiplicative watermark embedding parameter, the relationship between watermark embedded strength factor and watermarked image quality is developed through experiments with the peak signal-to-noise ratio (PSNR) and structural similarity index measure (SSIM), respectively. Secondly, to detect the watermark information, the generalized Gaussian distribution (GGD) has been utilized to model the contourlet coefficients. Furthermore, positions of the blocks selected, watermark embedding factor, and watermark size have been used as side information for watermark decoding. Finally, several experiments have been conducted on eight images, and the results prove the effectiveness of the proposed watermarking approach. Concretely, our watermarking method has good imperceptibility and strong robustness when against Gaussian noise, JPEG compression, scaling, rotation, median filtering, and Gaussian filtering attack.

\section{Introduction}

Transmitting and sharing digital multimedia have become more convenient with the rapid development of the network. However, such phenomenon results in security issues, such as authentication, copyright protection, and fingerprinting [1-7]. Digital watermarking can be used as an effective method to address these problems. Generally, in the watermarking process, some useful information (e.g., watermark data) is embedded into an original signal while ensuring its quality. Furthermore, robustness and imperceptibility are the main factors in digital image watermarking. Many image watermarking algorithms have been presented in the literature. On the basis of the embedding method, most algorithms can be divided into three categories, namely, additive, quantization, and multiplicationbased watermarking algorithms.

For the additive-embedding watermarking approach, the watermark information is directly added to the host image coefficients or image block of the same size. Generally, the coefficients can be obtained from some common transforms, including discrete wavelet transform (DWT), discrete cosine transform (DCT), and Fourier transform. The additiveembedding watermarking embeds the watermark information in the most important frequency domain of image perception, which is similar to the spread spectrum communication idea in the communication system. Cox et al. [8] first designed a digital watermarking method based on the idea of spread spectrum, which embedded watermark data in the important perception transformation coefficient of the host signal by applying the spread spectrum principle. Cox's spread spectrum watermarking algorithm has been considered a representative method. The only deficiency is that the digital watermarking algorithm requires participation of the original image when detecting watermark information, indicating that it is not a blind watermarking algorithm. Subsequently, Cheng et al. [9] proposed an additive watermarking approach, which detects the watermark by 
using the generalized Gaussian distribution (GGD). Experiments show that this distribution can effectively control the detection error probability of the watermark. Liu et al. [10] transformed the test signal into the DCT domain. Moreover, a local optimal detection model that is suitable for any host signal was derived by conducting hypothesis testing analysis in this domain. Although these methods $[9,10]$ can detect watermark information effectively, their parameter estimation process is complex. To address this problem, Kwitt et al. [11] proposed a lightweight blind optimal detector for additive watermarking; it is expected to be useful in resisting watermark desynchronization. Zhang et al. [12] proposed a high-security additive watermarking algorithm by utilizing gyrator transform and matrix decomposition. A key innovation of this algorithm is to adopt an invariant integer wavelet transform, which transforms the image wavelet coefficients into integers, thereby enhancing the performance of the watermarking.

In the quantization-based watermarking method, the main procedure is to embed the watermark data into the host signal by designing a corresponding quantizer. The watermark data are detected according to the quantization interval of the image transform coefficient to extract watermark. Many watermarking methods with quantization scheme have been proposed in recent years. Chen et al. proposed a digital watermarking method with the quantization index modulation (QIM) scheme; it is the most representative quantization watermarking algorithm based on edge information coding [13]. QIM has the characteristics of high capacity, blind detection, and simple implementation. However, QIM watermarking has two main shortcomings. First, it is sensitive to amplitude scaling attacks; second, it is not robust to gain attacks. Researchers proposed corresponding improvement methods to address these problems. In view of the sensitivity of the QIM watermarking method to scaling attacks, researchers mainly improved it in accordance with the quantization step size. To solve the inconsistency of quantization step between the embedded end and the receiver end, as well as the adaptability problem of quantization step, several watermarking methods have been proposed, such as rational dither modulation [14] and adaptive QIM [15]. Furthermore, to enhance the robustness of the QIM watermarking against gain attack, the quantization watermarking [16], sample projection-based quantization [17], P-norm ratio-based quantization [18], angle quantization [19], complex wavelet domain $l_{1}$ norm quantization [20], and random projectionbased quantization methods [21] have been proposed one after another. These quantization watermarking methods mainly aim to enable the watermark algorithm to obtain invariance to the scaling or gain attacks, and the watermark has strong robustness performance in resisting compression, filtering, and gain attacks. However, the performance of these quantization methods in desynchronization attacks is still inadequate. To further enhance the robustness of quantization watermarking, some researchers have designed corresponding quantization watermarking algorithms by combining the just noticeable distortion (JND) model, image texture complexity, and texture direction features, such as texture direction quantization [22], pair quantization based on extended JND [23, 24], and mixed modulation quantization using singular value decomposition [25]. These quantization methods are combined with image features; they can retain image orientation features and reduce image distortion after watermark embedding. However, these methods are generally vulnerable to noise attack.

The performance of the multiplicative embedding-based watermarking method is similar to that of the quantization watermarking method. The multiplicative watermarking algorithm is usually combined with the human visual perception model, and the embedded strength factor varies with the intensity of the original signal. Moreover, a good tradeoff between imperceptibility and robustness can be achieved in the multiplicative watermarking algorithm. Akhaee et al. [26] developed an image watermarking method based on a "scaling" strategy by using the Watson entropy visual masking. The watermark data were embedded into the image block with high entropy to improve the invisibility of the watermark. The algorithm is robust against Gaussian filtering, Gaussian noise, and scaling attacks. However, the entropy value of the image block changes after embedding the watermark; this finding is inconsistent with the entropy of the image block prior to embedding the watermark, thereby reducing the robustness of the watermark against synchronization attacks. Subsequently, Akhaee et al. [27] proposed a scaling-based image watermarking method with contourlet transform in a noisy environment. Experiments demonstrated that the robustness of this watermarking method is good. However, the algorithm has high complexity. Different from the Watson entropy visual masking, Khalilian et al. [28] proposed a multiplicative watermarking algorithm by taking advantage of the visual saliency model. They designed an adaptive embedding factor by combining visual saliency and texture masking. On the one hand, the embedding factor should increase with the distance from the significant region of the image. On the other hand, the watermark embedding strength should be larger in regions with rich texture. This method improves the robustness of the watermarking when against some common image processing attacks. However, the performance of their watermarking still needs to be enhanced in terms of resisting antidesynchronization attack. Moreover, some visual attention-based watermarking methods have been presented in the last few years. For example, Bhowmik et al. [29] embedded high-strength and low-strength watermarks into significant and insignificant regions of vision, respectively, thereby improving the watermarking performance. Hernandez et al. [30] proposed a video watermarking algorithm that took full advantage of the video's spatiotemporal characteristics and minimized the perceived redundancy of the video. Thus, the trade-off between imperceptibility and robustness has been achieved in their method. Yadav et al. [31] developed an image watermarking algorithm by using an adaptive embedded factor, which only used image variance information to compute watermark embedded factor. However, the performance of this method is weak when resisting rotation attacks. 
Inspired by literature [28], an image watermarking algorithm was developed based on the visual saliency model in the contourlet transform domain. The main contributions of our work are summarized as follows:

(1) An adaptive watermark embedded strength factor is exploited with a visual saliency model, which can achieve a good trade-off between the robustness and imperceptibility of the watermarking.

(2) The watermark information was embedded into the contourlet coefficients with high energy that can enhance the imperceptibility of watermarking.

The remainder of this paper is organized as follows. The belief concept of the contourlet transform is introduced in Section 2. Section 3 introduces the proposed watermark embedding and detection method. Section 4 shows the experimental results of the proposed watermarking and the comparative results with other watermarking approaches. Finally, the conclusions are summarized in Section 5.

\section{Brief Introduction of Contourlet Transform}

In 2005, Do et al. [32] proposed a "real" 2D representation of images, that is, the contourlet transform. It captures the segmented conic curves of an image by using different subband scales and frequencies, which have directivity and anisotropy, thereby enabling the contourlet transform to obtain a "sparser" representation. Thus, the contourlet transform has the characteristics of sparse representation at both spatial and directional resolutions. In contourlet transform, multiscale and directional analyses are performed separately. First, the image was transformed into one coarse version plus a set of band-pass images by the Laplacian pyramid (LP) method. Second, each LP band-pass image was decomposed into a number of subbands with 2D quincunx filtering and critical subsampling. Therefore, the contourlet transform can decompose images into multidirectional subbands at multiple scales. Figure 1 illustrates a diagram of the contourlet transform. Furthermore, we have utilized the contourlet toolbox to decompose the "Peppers" image [32]. Figure 2 shows the result of applying the contourlet transform on the "Peppers" image. The figure clearly shows that the contourlet transform can decompose the "Peppers" image into multidirectional subbands.

\section{Watermark Embedding and Decoding}

In this section, Figure 3 shows the proposed watermark embedding and watermark detection procedure. As shown in Figure 3, we embed the watermark data into the contourlet coefficients with high energy in our implementation. In addition, we utilize the visual saliency model to construct the watermark embedded strength factor; thus, a trade-off between the invisibility and robustness of the watermark can be achieved elegantly with the watermark embedded strength factor. In the watermark detection stage, we model the contourlet coefficients with GGD to detect the watermark due to the non-Gaussian property of the contourlet coefficients.

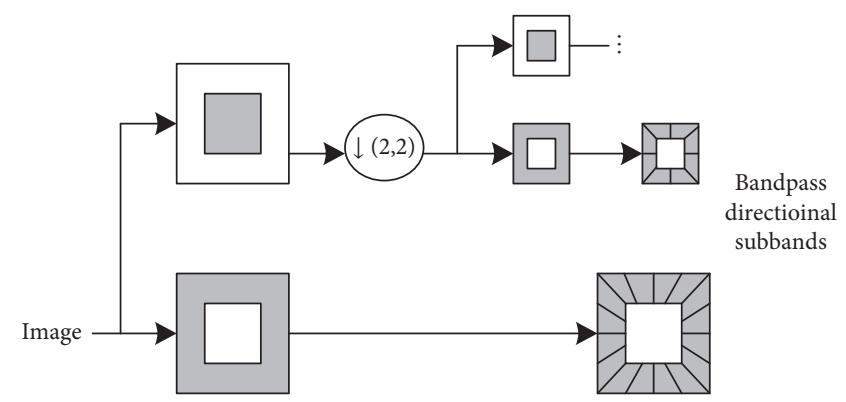

Figure 1: The diagram of the contourlet transform.

3.1. Proposed Watermark Embedding. The procedure of the proposed watermark embedding in Figure 3(a) can be generalized as follows:

Step 1: We segment the host image into $L \times L$ blocks and select the first $N$ image blocks with high energy. The energy is calculated as the sum of the squares of the absolute values of the pixels of the image block. Consequently, the energy of block [28] can be computed by $E=\sum_{m=1}^{M} \sum_{n=1}^{N}\|B(m, n)\|^{2}$, where $M \times N$ denotes the size of the image block $B$ and $(m, n)$ represents the positions of image block. Generally, a larger value of the energy of image block implies that this image region contains more important coefficients and should be considered a significant image block in comparison with other image blocks. Therefore, to improve the robustness of the watermarking, the watermark is embedded into the image blocks with high energy.

Step 2: Then, we decompose each selected image block by using a two-level contourlet transform. Thus, we embed the watermark data into the coefficients of lowfrequency subband. The host contourlet coefficient vectors are denoted as $x=\left[x_{1}, x_{2}, \ldots, x_{n}\right]$, and the watermarked contourlet coefficient vectors are denoted as $y=\left[y_{1}, y_{2}, \ldots, y_{n}\right]$. Suppose that the watermark is $w=\left[w_{1}, w_{2}, \ldots, w_{n}\right] \quad$ with $n$ components and $w_{i} \in\{-1,1\}$; the watermark embedding process can be expressed as follows:

$$
y=x(1+\alpha w),
$$

where $\alpha$ denotes the embedded strength factor and its value was calculated in Section 3.2.

Step 3: Repeat Step 2 for each image block.

Step 4: Two-level inverse contourlet transform on the watermarked image subband is performed, and it is combined with the image subbands, which are not embedded watermark information, to obtain the whole watermarked image.

3.2. Watermark Embedded Strength Factor. The JND threshold has been widely applied in the field of image processing. Its value is often higher in the image texture region [33]. On the basis of [33], the image texture region 

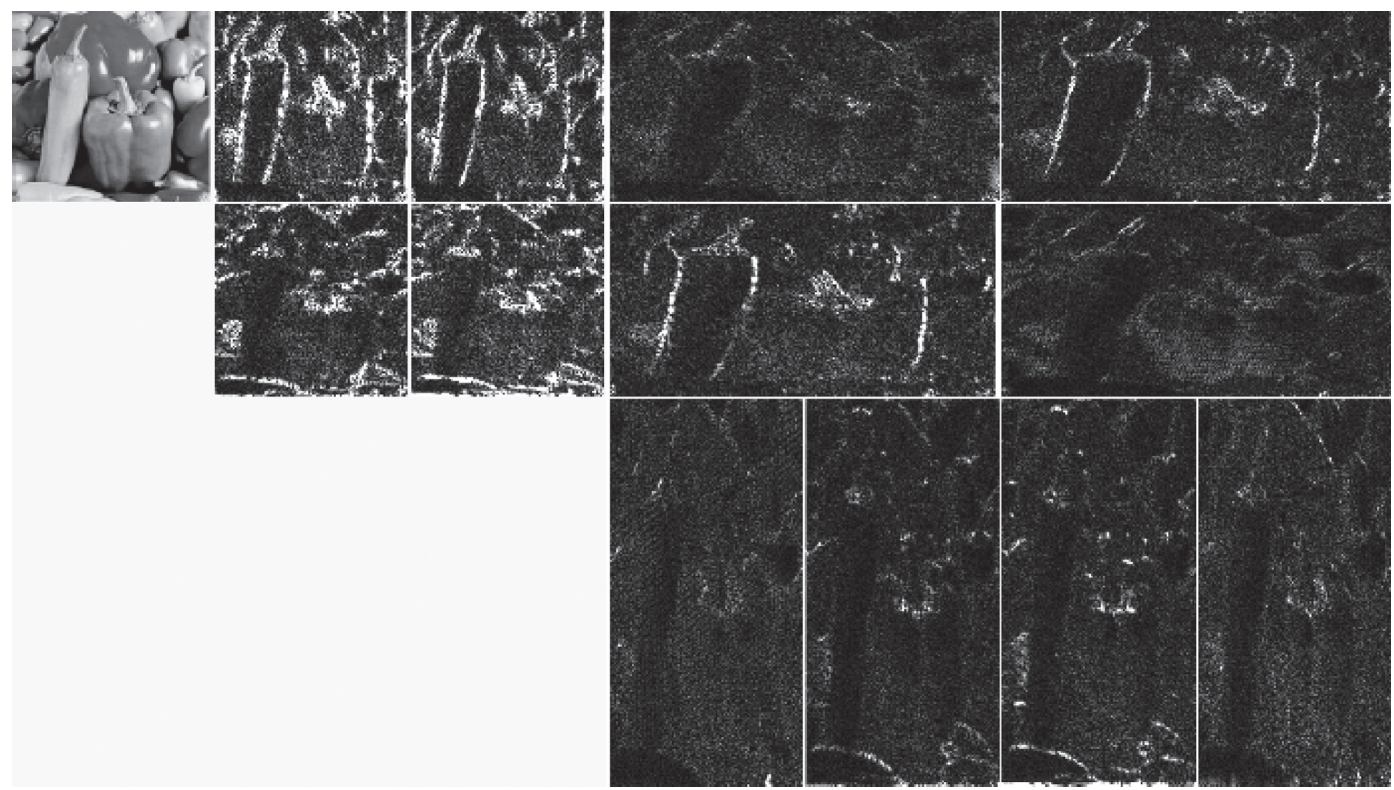

Figure 2: Contourlet transform of the "Peppers" image using two levels [32].

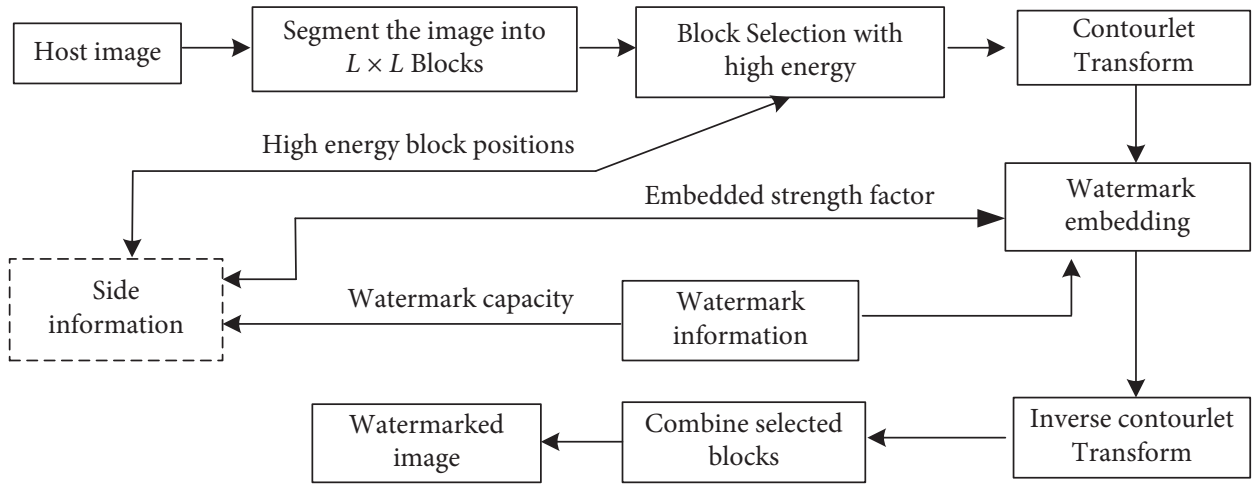

(a)

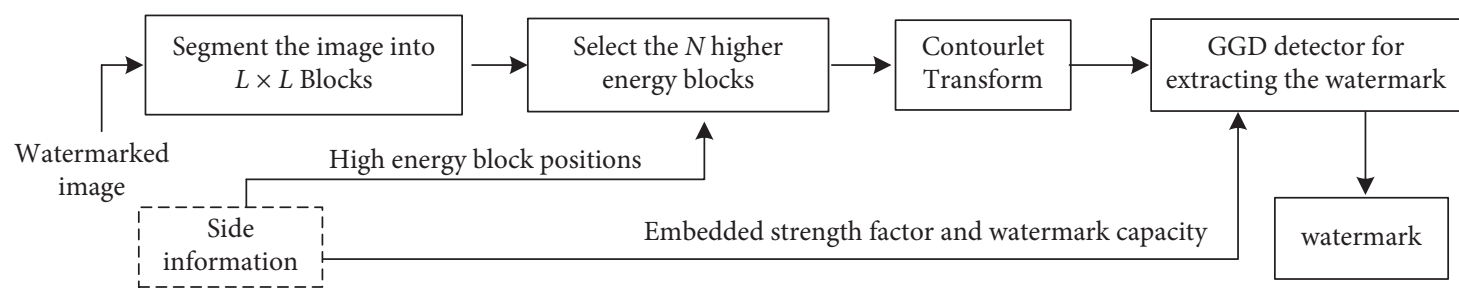

(b)

FIGURE 3: Block diagram of the proposed method. (a) Watermark embedding. (b) Watermark detection.

can hide more information without being perceived by human eyes. Therefore, the embedded strength factor can select a high value. Literature [27] used this fact to develop an image watermarking algorithm. Literature [34] shows that the human visual system tends to focus on the salient areas of an image. As a result, the image salient area hides more distortion, and the embedded strength factor can be enhanced. Therefore, to calculate the embedded strength factor, we take advantage of the texture masking and visual saliency model in this study. The calculation process is summarized as follows.

First, we use a two-level contourlet transform to decompose the host image, which obtains a low-frequency subband, four subbands, and eight subbands from the coarsest scale to the finest scale (Figure 2). Therefore, we compute the energy of directional subband of each block according to the property of the image texture masking. The calculation can be expressed as follows: 


$$
E_{H}=\sum_{i=1}^{12} E_{H_{i}}
$$

where $E_{H_{i}}$ is the $i$-th directional subband's energy of each image block. Each image block has 12 directional subbands after the two-level decomposition with contourlet transform. Suppose that $\widetilde{E}_{H}$ denotes the average energy of twelve image blocks. When increasing the average energy, the watermark embedded strength factor could increase correspondingly. Hence, according to [27], the watermark embedded strength factor of the high-frequency part can be written as follows:

$$
\alpha_{H F}=\eta-\rho \cdot e^{-\zeta \cdot \widetilde{E}_{H}},
$$

where $\eta, \rho$, and $\zeta$ are set to $1.025,0.02$, and $\times$, respectively. These parameters are determined by experimental simulation. In the right part of equation (3), for $\alpha_{\mathrm{HF}}$, the parameter $\eta$ is set to 1.025 for larger image energy $\widetilde{E}_{H}$, when the exponential function vanishes. This parameter is set to 1.025 in our experiments mainly because it maintains the imperceptibility of the image when used in high image energy when the exponential term disappears. On the contrary, for small image energy, we set parameter $\rho$ to 0.02 . Parameter $\zeta$ has an important effect in the increasing rate of watermark embedded strength factor; its value is set to $\times$ mainly because it can achieve a good trade-off between the robustness and imperceptibility of the watermarking. Therefore, the parameter setting of the watermark embedded strength factor is mainly based on the size of image energy. The main reason is to embed the watermark information while maintaining the imperceptibility of the image watermark.

Then, inspired by [35], we modified the embedded strength factor, which is denoted by $\alpha_{\mathrm{HF}}$ by applying visual saliency. Suppose that $D_{S}$ represents the saliency distance of each block and $D_{s}^{\max }$ denotes the maximum saliency distance in all image blocks. Therefore, the watermark embedded strength factor can be expressed by $1+0.02 / D_{s}^{\max } D_{s}$. Finally, the modified watermark embedded strength factor can be represented as follows:

$$
\begin{aligned}
\alpha & =\alpha_{\mathrm{HF}} \times\left(1+\frac{0.02}{D_{s}^{\max }} D_{s}\right)-1.0 \\
& =\left(\eta-\rho \cdot e^{-\zeta \cdot \widetilde{E}_{H}}\right) \times\left(1+\frac{0.02}{D_{s}^{\max }} D_{s}\right)-1.0 .
\end{aligned}
$$

3.3. Watermark Decoding. In this section, we model the contourlet coefficients by the GGD. The probability density function of the GGD model is represented as follows:

$$
p_{X}(x)=A e^{\left(-(\beta|x-\mu|)^{c}\right)},
$$

where $\quad A=\beta c / 2 \Gamma(1 / c), B=1 / \sigma(\Gamma(3 / c) / \Gamma(1 / c))^{1 / 2}, \quad$ and $\mu, \sigma$ denote the mean value and variance, respectively. $\Gamma(\cdot)$ is the gamma function when $\Gamma(z)=\int_{0}^{\infty} e^{-t} t^{z-1} \mathrm{~d} t, z>0$, and $c$ denotes the shape parameter. Watermark detection can perform the detection and evaluation of signals. The hypothesis test can be drawn as follows, using the likelihood ratio test (LRT):

$$
\left\{\begin{array}{l}
H_{0}: \alpha=0 \text { (no watermark) } \\
H_{1}: \alpha>0 \text { (watermark) }
\end{array}\right.
$$

where $H_{0}$ and $H_{1}$ are the null and alternative hypotheses. According to the statistical signal processing method, the maximum likelihood ratio can be represented as follows:

$$
l(y)=\frac{p\left(y \mid H_{1}\right)}{p\left(y \mid H_{0}\right)} \approx \frac{p\left(y \mid H_{1}\right)}{p(y \mid 0)} .
$$

Proofs of (7) are as follows [36]:

$$
\int_{-1}^{1} P_{y}\left(y_{i} \mid w_{i}\right) \mathrm{d} w_{i}=\int_{-1}^{1} \frac{1}{1+\alpha_{i} w_{i}} \times P_{x}\left(\frac{y_{i}}{1+\alpha_{i} w_{i}}\right) \mathrm{d} w_{i} \text {. }
$$

Let $t=y_{i} /\left(1+\alpha_{i} w_{i}\right)$. Then, the integrand substitutes $t$ for $w_{i}$; (8) can be rewritten as follows:

$$
\int_{-1}^{1} P_{y}\left(y_{i} \mid w_{i}\right) d w_{i}=\int_{y_{i} /\left(1+\alpha_{i}\right)}^{y_{i} /\left(1-\alpha_{i}\right)} \frac{1}{\lambda_{i} t} \times P_{x}(t) \mathrm{d} t
$$

One order Taylor series of $\left(1 / \alpha_{i} t P_{x}(t)\right)$ around $y_{i}$ is expanded as follows:

$$
\frac{1}{\alpha_{i} t} P_{x}(t)=\frac{1}{\alpha_{i} y_{i}} P_{x}\left(y_{i}\right)+\left.\frac{d}{\mathrm{~d} t}\left(\frac{1}{\alpha_{i} t} P_{x}(t)\right)\right|_{t=y_{i}}\left(t-y_{i}\right) \text {. }
$$

Therefore, (10) is rewritten as follows:

$$
\int_{-1}^{1} P_{y}\left(y_{i} \mid w_{i}\right) \mathrm{d} w_{i}=\int_{y_{i} /\left(1+\alpha_{i}\right)}^{y_{i} /\left(1-\alpha_{i}\right)} \frac{1}{\lambda_{i} y_{i}} P_{x}\left(y_{i}\right) \mathrm{d} t+\left.\frac{d}{\mathrm{~d} t}\left(\frac{1}{\alpha_{i} t} P_{x}(t)\right)\right|_{t=y_{i}} \int_{y_{i} /\left(1+\alpha_{i}\right)}^{y_{i} /\left(1-\alpha_{i}\right)}\left(t-y_{i}\right) \mathrm{d} t
$$

$\alpha_{i} \ll 1, \mathrm{~d} /\left.\mathrm{d} t\left(1 / \alpha_{i} t P_{x}(t)\right)\right|_{t=y_{i}} \int_{y_{i} /\left(1+\alpha_{i}\right)}^{y_{i} /\left(1-\alpha_{i}\right)}\left(t-y_{i}\right) \mathrm{d} t$ is approximately zero in (11). Therefore, equation (11) can be further expressed as follows: 


$$
\int_{-1}^{1} P_{y}\left(y_{i} \mid w_{i}\right) \mathrm{d} w_{i} \approx \int_{y_{i} /\left(1+\alpha_{i}\right)}^{y_{i} /\left(1-\alpha_{i}\right)} \frac{1}{\alpha_{i} y_{i}} P_{x}\left(y_{i}\right) \mathrm{d} t=\frac{1}{\alpha_{i} y_{i}} P_{x}\left(y_{i}\right) \frac{2 \alpha_{i} y_{i}}{1-\alpha_{i}^{2}}=2 P_{x}\left(y_{i}\right) .
$$

Therefore, $P\left(y \mid H_{0}\right) \approx 1 / 2^{N} \prod_{i=1}^{N}\left(2 P_{x}\left(y_{i}\right)\right)=P(y \mid 0)$.

On the basis of the analysis, we can rewrite the maximum likelihood ratio by combining watermark embedding (1) and the GGD model as follows:

$$
l(y)=\ln \frac{P\left(y \mid H_{1}\right)}{P\left(y \mid H_{0}\right)} \approx \ln \frac{P\left(y \mid H_{1}\right)}{P(y \mid 0)}=\ln \frac{\prod_{i=1}^{N}\left(A / 1+\alpha_{i} w_{i} \exp \left(-\left|\beta_{i} y_{i} / 1+\alpha_{i} w_{i}\right|^{c}\right)\right)}{\prod_{i=1}^{N}\left(A \exp \left(\left.\dashv \beta_{i} y_{i}\right|^{c}\right)\right) .}
$$

Furthermore, equation (13) can be simply represented as follows:

$$
l(y)=\sum_{i=1}^{N}\left(-\alpha_{i} w_{i}+c|\beta y|^{c} \alpha_{i} w_{i}\right)
$$

Thus, we can write the watermark detector as follows:

$$
T(y)=\frac{\partial l(y)}{\partial \alpha_{i}}=\sum_{i=1}^{N}\left(-w_{i}+c\left|\beta y_{i}\right|^{c} w_{i}\right)
$$

Next, we can compute the watermark detection threshold. The Gaussian distribution characteristic of the watermark detector under the null hypothesis condition and its mean is zero. As a result, we can calculate the watermark detection threshold as follows:

$$
\tau=\sigma_{T} Q^{-1}\left(P_{f}\right),
$$

where $\tau$ denotes the watermark detection threshold, $\sigma_{T}=\sqrt{\sum_{i=1}^{N} c w_{i}^{2}}$ represents the variance, and $Q(x)=(1 / \sqrt{2 \pi}) \int_{x}^{+\infty} \exp \left(-t^{2} / 2\right) \mathrm{d} t$ denotes the right-tail probability of the Gaussian distribution. $P_{f}=P\left(T(y)>\tau \mid H_{0}\right)=Q\left(\tau / \sigma_{T}\right)$ represents the false alarm probability.

Generally, false alarm is generated due to the existence of the watermark information detected in the unwatermarked image. A missed alarm is the phenomenon in which the watermark detector does not detect the watermark information in the watermarked image. Therefore, the receiver operating characteristic (ROC) curve of the watermarking can be derived as follows.

Suppose $P_{0}$ denotes the detection probability of watermark. Hence, $\left(1-P_{0}\right)$ can represent the missed alarm probability. According to the statistical hypotheses and the central limit theorem, the mean and variance of the distribution of the host image and watermarked image can be estimated; they are denoted as $\mu_{T_{0}}, \mu_{T_{1}}$ and $\sigma_{T_{0}}, \sigma_{T_{1}}$, respectively. As a result, $P_{0}$ can be written as follows:

$$
P_{d}=Q\left(\frac{\sigma_{T_{0}} Q^{-1}\left(P_{f}\right)+\mu_{T_{0}}-\mu_{T_{1}}}{\sigma_{T_{1}}}\right),
$$

where $\sigma_{T_{0}} \approx \sigma_{T_{1}}, \mu_{T_{0}}=0$, and $\mu_{T_{1}}=\left(1 / N \sum_{i=1}^{N} \alpha_{i}\right) \cdot \sigma_{T_{0}}^{2}$. Let $\mathrm{SNR}=\mu_{T_{1}} / \sigma_{T_{1}}=\left(1 / N \sum_{i=1}^{N} \alpha_{i}\right) \cdot \sigma_{T_{0}}$.
Finally, the ROC relationship can be defined as follows:

$$
P_{d}=Q\left(Q^{-1}\left(P_{f}\right)-\mathrm{SNR}\right) .
$$

\section{Experimental Results}

In this regard, to verify the effectiveness of the proposed watermarking method, several experiments have been performed, including the imperceptibility, robustness, and performance of watermark detection. We have compared the proposed watermarking with other related watermarking approaches. All experiments have been performed on a PC with $4.0 \mathrm{GHz}$ Intel Core i7 $\mathrm{CPU}$ and $16 \mathrm{G}$ RAM. The simulation software was MATLAB R2018a that ran in 64-bit Windows 10. In summary, the simulation settings are provided in Table 1.

4.1. Imperceptibility Test. We have tested eight standard images, which include Lena, Barbara, Bridge, Boat, Elaine, Mandrill, Peppers, and Man, to demonstrate the invisibility of the proposed method; the size of each standard image is $512 \times 512$. In our implementation, a two-level contourlet transform has been applied to decompose each image block. The filters are set to "Pivka." Figure 4 only shows the host images and their watermarked version made by applying our method with $16 \times 16$ blocks and a 512-bit watermark capacity due to the limited space. Figure 4 shows that the imperceptibility of our method is satisfied. Therefore, finding the difference between the original image and their watermarked version is difficult.

In addition, the embedded strength factor can be adapted and adjusted according to the watermark capacity to further enhance the performance of the proposed method. The relationship between the embedded parameter and watermarked image quality is developed through experiments, and the results are shown in Figures 5 and 6 . The performance is mainly measured by peak signal-tonoise ratio (PSNR) and structural similarity index measure (SSIM) [37]. As shown in Figures 5 and 6, when the watermark embedded strength factor increases, the values of PSNR and SSIM decrease. The range of embedded strength factor can be set within 0.005 to 0.025 to balance the imperceptibility, robustness, and watermark capacity of watermarking. 
TABLE 1: Experimental parameter settings.

\begin{tabular}{lc}
\hline Parameter name & Configuration \\
\hline Experimental platform & Window 10, MATLAB R2018a \\
Test images & Lena, Barbara, Bridge, Boat, Elaine, Mandrill, Peppers, and Man \\
Image size & $512 \times 512$ \\
Wavelet filters of contourlet transform & Pivka \\
Watermark length (bits) & 512 \\
Decomposition level & Two-level \\
Performance evaluation & PSNR, SSIM, and bit error rate (BER) \\
\hline
\end{tabular}
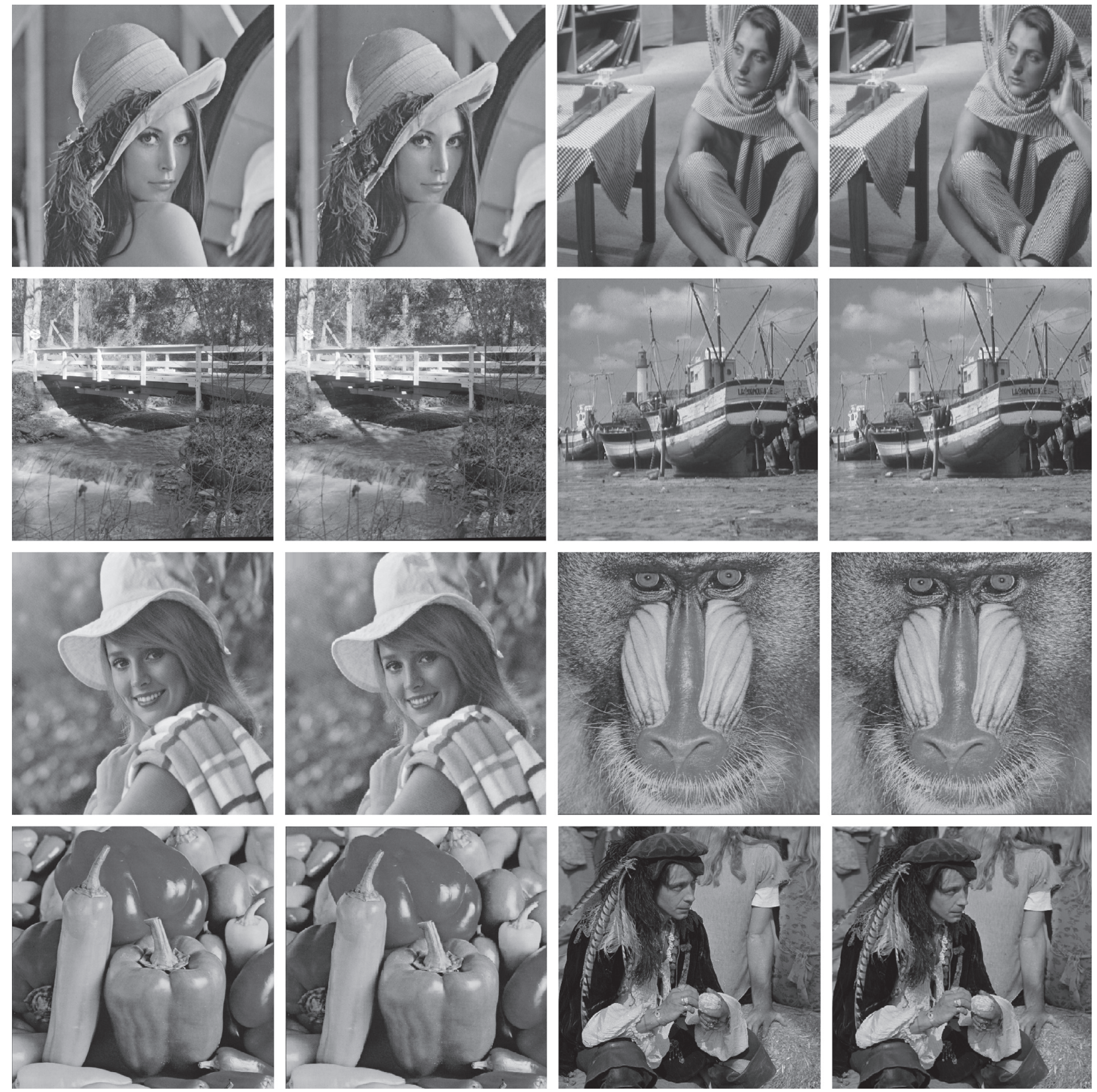

Figure 4: Original and watermarked versions: Lena, Barbara, Bridge, Boat, Elaine, Mandrill, Peppers, and Man. For each image, the left and right parts denote the original image and the watermarked image, respectively. 


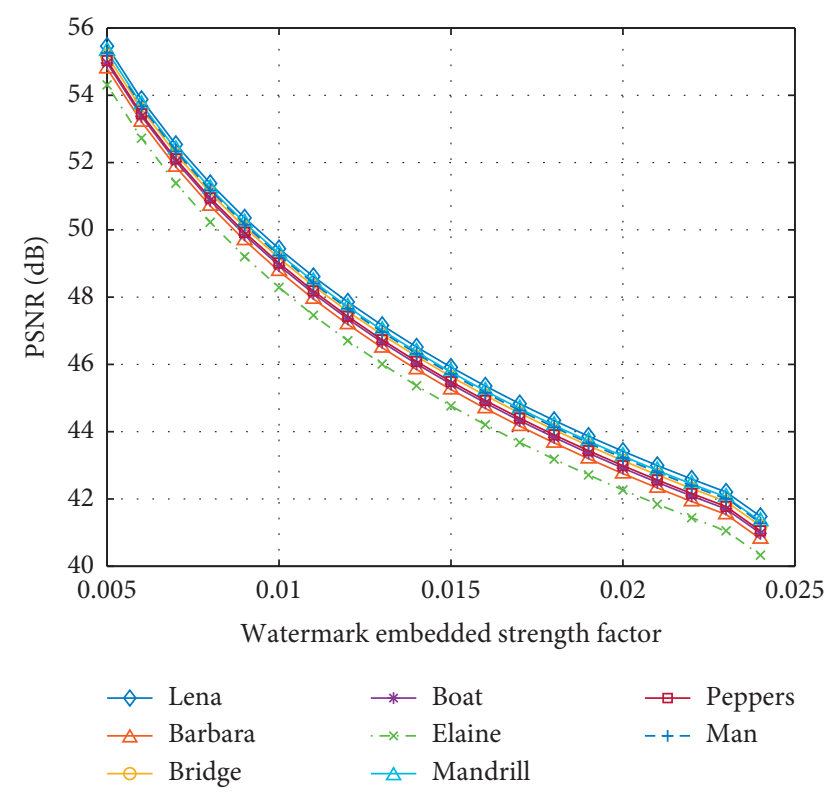

FIGURE 5: PSNR versus watermark embedded strength factor with watermark capacity 2048.

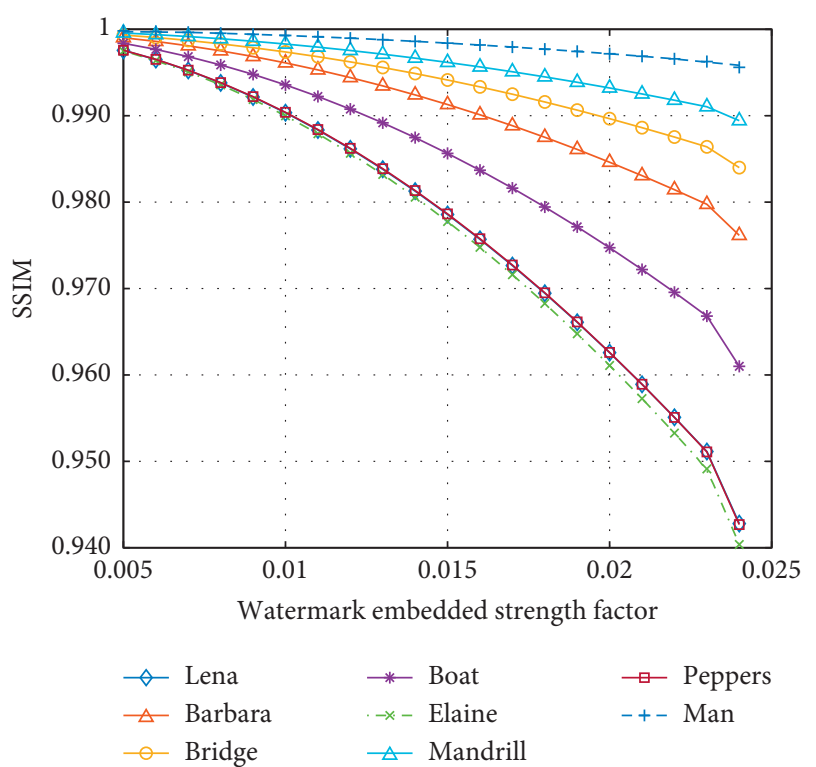

FIGURE 6: SSIM versus watermark embedded strength factor with watermark capacity 2048.

4.2. Robustness Test. In this section, to assess the robustness of the proposed watermarking, several experiments have been performed in common image processing and some geometric attacks. These attacks include additive whiten Gaussian noise, salt and pepper noise, median filtering, rotation, cropping, flipping, scaling, JPEG compression, and Gaussian filtering attack. Furthermore, to evaluate the effectiveness of our watermarking method, we have compared it with other related watermarking approaches, which include the methods in $[27,31]$ and [38]. Moreover, the robustness performance is measured through the bit error rate (BER).

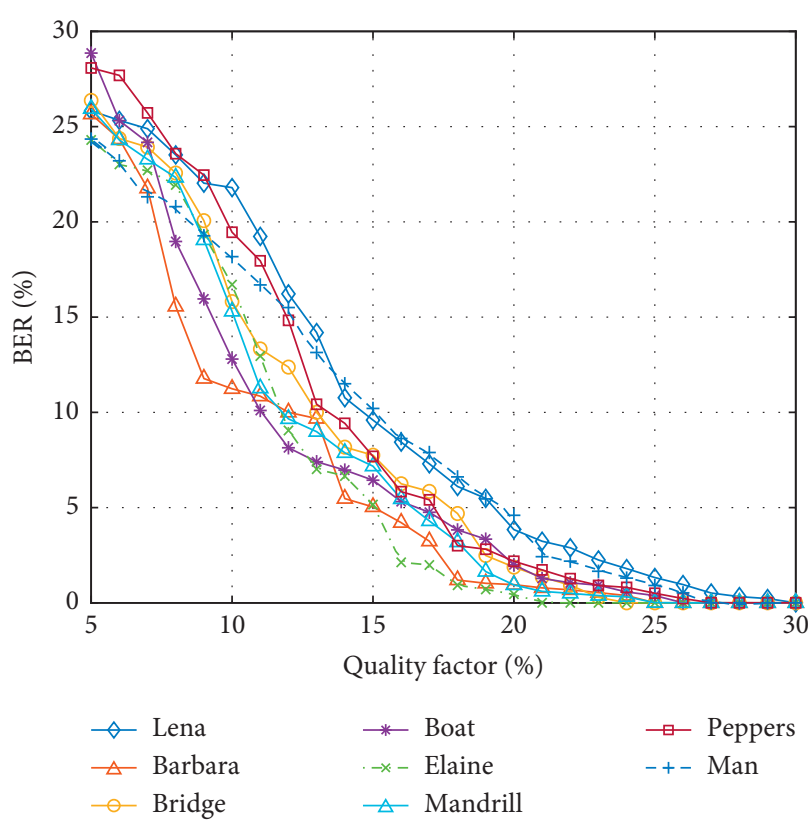

FIGURE 7: BER (\%) results under JPEG compression attack.

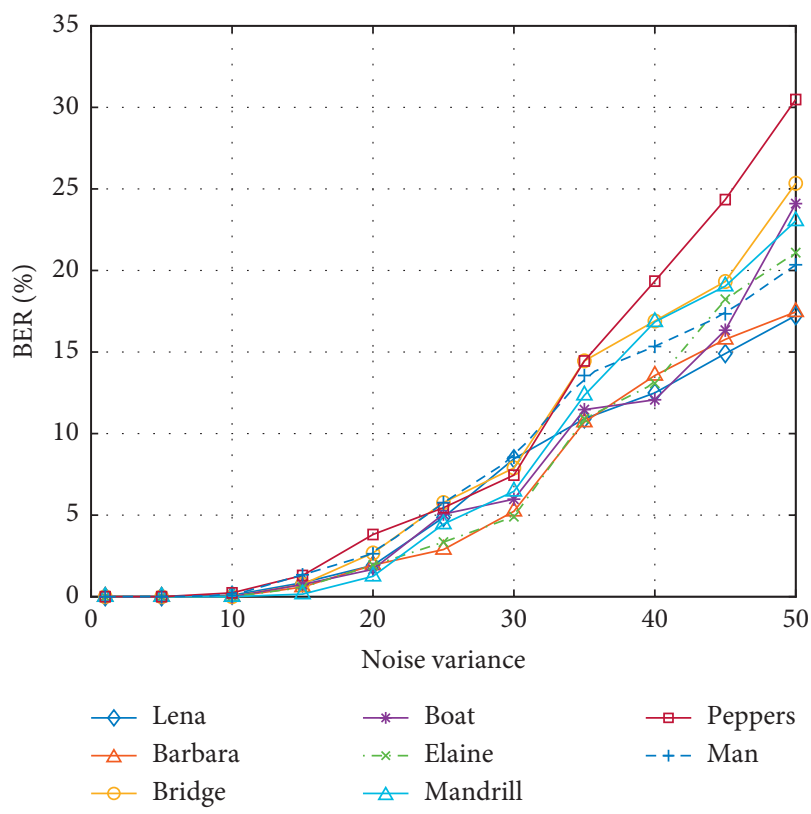

Figure 8: BER (\%) results under Gaussian noise attack.

We have performed two common experiments under JPEG compression and Gaussian noise attack. The result is shown in Figures 7 and 8. In this work, the watermark capacity is 512 bits. Figure 7 shows that our method has satisfying robustness on JPEG compression attack. Similarly, Figure 8 shows that our watermarking method has good robustness against Gaussian noise attacks. Moreover, Tables 2 and 3 show the comparison of the performance of our method with other methods under common image processing, geometric, and combined attacks. All watermarking methods, for the purpose of comparison, use the same watermark capacity. The watermark capacity of all methods is also 512 bits in Tables 2 and 3 . 
TABLE 2: BER (\%) results of various watermarking methods under common attacks.

\begin{tabular}{|c|c|c|c|c|c|c|}
\hline Image & Methods & Gaus.noi. 20 & Salt.Pep.0.05 & JPEG 20\% & Gau.filt. $3 \times 3$ & Med.filt. $3 \times 3$ \\
\hline \multirow{4}{*}{ Lena } & Method [27] & 3.02 & 3.43 & 5.63 & - & - \\
\hline & Method [31] & 2.41 & 13.78 & 9.24 & 1.19 & 1.56 \\
\hline & Method [38] & 6.29 & - & 20.76 & 1.83 & 4.95 \\
\hline & Proposed & 2.25 & 11.24 & 4.83 & 2.86 & 3.72 \\
\hline \multirow{4}{*}{ Barbara } & Method [27] & 2.56 & 3.95 & 2.14 & - & - \\
\hline & Method [31] & 1.89 & 10.26 & 8.30 & 0.82 & 1.65 \\
\hline & Method [38] & 7.34 & - & 18.85 & 1.49 & 5.36 \\
\hline & Proposed & 2.08 & 5.23 & 2.35 & 1.73 & 2.20 \\
\hline \multirow{4}{*}{ Bridge } & Method [27] & 2.94 & 9.44 & 6.79 & - & - \\
\hline & Method [31] & 3.81 & 12.50 & 8.96 & 2.49 & 2.16 \\
\hline & Method [38] & 6.93 & - & 19.50 & 1.68 & 5.18 \\
\hline & Proposed & 2.59 & 9.69 & 2.61 & 3.80 & 5.43 \\
\hline \multirow{4}{*}{ Boat } & Method [27] & 3.12 & 10.17 & 5.29 & - & - \\
\hline & Method [31] & 4.93 & 14.98 & 7.42 & 1.85 & 3.26 \\
\hline & Method [38] & 6.68 & - & 18.20 & 1.56 & 5.64 \\
\hline & Proposed & 2.34 & 12.05 & 2.58 & 2.23 & 6.69 \\
\hline \multirow{4}{*}{ Elaine } & Method [27] & 2.59 & 13.30 & 3.68 & - & - \\
\hline & Method [31] & 2.08 & 15.19 & 5.89 & 2.79 & 4.92 \\
\hline & Method [38] & 6.34 & - & 17.35 & 2.76 & 5.54 \\
\hline & Proposed & 2.13 & 14.58 & 0.87 & 2.55 & 6.80 \\
\hline \multirow{4}{*}{ Mandrill } & Method [27] & 1.78 & 4.87 & 3.22 & - & - \\
\hline & Method [31] & 2.34 & 10.48 & 6.45 & 1.38 & 2.64 \\
\hline & Method [38] & 5.95 & - & 18.29 & 1.82 & 5.97 \\
\hline & Proposed & 1.82 & 7.67 & 1.33 & 2.19 & 4.50 \\
\hline \multirow{4}{*}{ Peppers } & Method [27] & 5.70 & 12.79 & 6.86 & - & - \\
\hline & Method [31] & 4.48 & 15.24 & 5.53 & 2.64 & 1.75 \\
\hline & Method [38] & 8.60 & - & 19.42 & 1.76 & 6.08 \\
\hline & Proposed & 4.27 & 12.87 & 2.73 & 2.39 & 1.44 \\
\hline \multirow{4}{*}{ Man } & Method [27] & 4.39 & 13.02 & 8.46 & - & - \\
\hline & Method [31] & 3.66 & 10.82 & 11.25 & 4.89 & 6.88 \\
\hline & Method [38] & 7.08 & - & 22.07 & 2.10 & 5.83 \\
\hline & Proposed & 2.45 & 11.77 & 4.94 & 4.75 & 5.32 \\
\hline
\end{tabular}

TABLE 3: BER (\%) results of various watermarking methods under geometric attacks.

\begin{tabular}{|c|c|c|c|c|c|}
\hline Image & Methods & Rot. $10^{\circ}$ & Scal. 0.75 & Crop.50\% & Rot.5+Scal .5 \\
\hline \multirow{4}{*}{ Lena } & Method [27] & 10.22 & 27.34 & 29.45 & 30.24 \\
\hline & Method [31] & 12.76 & 22.08 & 26.17 & 29.46 \\
\hline & Method [38] & 17.49 & 20.89 & 22.32 & 34.58 \\
\hline & Proposed & 9.68 & 19.97 & 20.80 & 21.73 \\
\hline \multirow{4}{*}{ Barbara } & Method [27] & 9.34 & 32.29 & 30.13 & 28.71 \\
\hline & Method [31] & 11.92 & 27.44 & 26.49 & 25.82 \\
\hline & Method [38] & 18.24 & 24.12 & 25.69 & 37.61 \\
\hline & Proposed & 6.60 & 26.80 & 16.32 & 20.94 \\
\hline \multirow{4}{*}{ Bridge } & Method [27] & 7.38 & 9.65 & 32.74 & 31.18 \\
\hline & Method [31] & 17.82 & 19.54 & 25.58 & 24.19 \\
\hline & Method [38] & 19.23 & 23.47 & 27.48 & 39.69 \\
\hline & Proposed & 9.56 & 16.89 & 24.22 & 23.71 \\
\hline \multirow{4}{*}{ Boat } & Method [27] & 9.51 & 18.36 & 28.67 & 28.40 \\
\hline & Method [31] & 8.84 & 24.07 & 20.34 & 29.33 \\
\hline & Method [38] & 15.02 & 28.73 & 26.85 & 38.87 \\
\hline & Proposed & 5.97 & 25.99 & 22.76 & 22.25 \\
\hline \multirow[t]{4}{*}{ Elaine } & Method [27] & 11.35 & 21.43 & 27.50 & 27.42 \\
\hline & Method [31] & 16.24 & 28.30 & 19.38 & 25.78 \\
\hline & Method [38] & 20.43 & 35.66 & 21.80 & 35.93 \\
\hline & Proposed & 10.33 & 30.12 & 16.14 & 25.39 \\
\hline Mandrill & Method [27] & 8.98 & 12.46 & 24.55 & 27.16 \\
\hline
\end{tabular}


TABLE 3: Continued.

\begin{tabular}{|c|c|c|c|c|c|}
\hline Image & Methods & Rot. $10^{\circ}$ & Scal. 0.75 & Crop.50\% & Rot.5+Scal .5 \\
\hline & Method [31] & 7.22 & 20.79 & 20.18 & 24.23 \\
\hline & Method [38] & 22.06 & 29.78 & 24.57 & 36.94 \\
\hline & Proposed & 6.50 & 17.43 & 12.84 & 19.59 \\
\hline \multirow[t]{4}{*}{ Peppers } & Method [27] & 12.45 & 23.78 & 29.43 & 29.68 \\
\hline & Method [31] & 13.39 & 18.76 & 21.80 & 30.49 \\
\hline & Method [38] & 18.87 & 27.26 & 23.18 & 38.68 \\
\hline & Proposed & 6.07 & 19.59 & 13.67 & 23.67 \\
\hline \multirow[t]{4}{*}{ Man } & Method [27] & 9.94 & 14.41 & 27.86 & 27.45 \\
\hline & Method [31] & 10.18 & 21.78 & 19.44 & 22.97 \\
\hline & Method [38] & 21.50 & 26.15 & 23.07 & 36.34 \\
\hline & Proposed & 7.83 & 20.42 & 10.29 & 21.80 \\
\hline
\end{tabular}

Table 2 shows the results of the simulation experiments under common image processing attacks, which cover Gaussian noise with noise variance 20, Salt and Pepper noise with noise variance of 0.05 , JPEG compression with a quality factor of $20 \%$, Gaussian filtering with the windows of size $3 \times 3$, and median filtering with the windows of size $5 \times 5$. Table 3 shows the results of the simulation experiments under geometric attacks, including the rotation attack with $10^{\circ}$ angle, amplitude scaling attack with factor 0.75 , cropping with factor $50 \%$, combination attack with rotation of $5^{\circ}$ angle, and scaling with a factor of 0.50 .

As shown in Tables 2 and 3, the proposed watermarking method has slightly better performance than the image watermarking methods. This finding is mainly due to the application of the following factors. First, we embed the watermark information into the image blocks with high energy in the contourlet transform domain. Second, the watermark embedded strength factor was constructed by taking advantage of the visual saliency model and texture masking. Thus, embedding the watermark can be adapted. As such, a good trade-off between the invisibility and robustness of the watermark can be achieved. Finally, the watermark detection performance can be improved by the GGD model.

However, the proposed watermark detector relies on partial original image feature information, such as positions of image blocks; thus, the proposed algorithm becomes semiblind. In the subsequent work, we will design a blind watermarking method.

4.3. Performance of Watermark Detection. The GGD is used to model the contourlet coefficients to further demonstrate the detection performance, and the ROC is utilized to measure the performance of watermark detection according to equation (18) of Section 3.3. Figure 9 shows the results and indicates that the detection performance of our method is satisfied. The main reason is that the contourlet coefficient distribution is highly nonlinear, and the GGD fits the contourlet coefficient effectively.

However, the proposed watermarking method performs weakly when resisting other attacks, including combinational attack amplitude scaling and JPEG compression, Salt and Pepper and Gaussian noise, and global affine

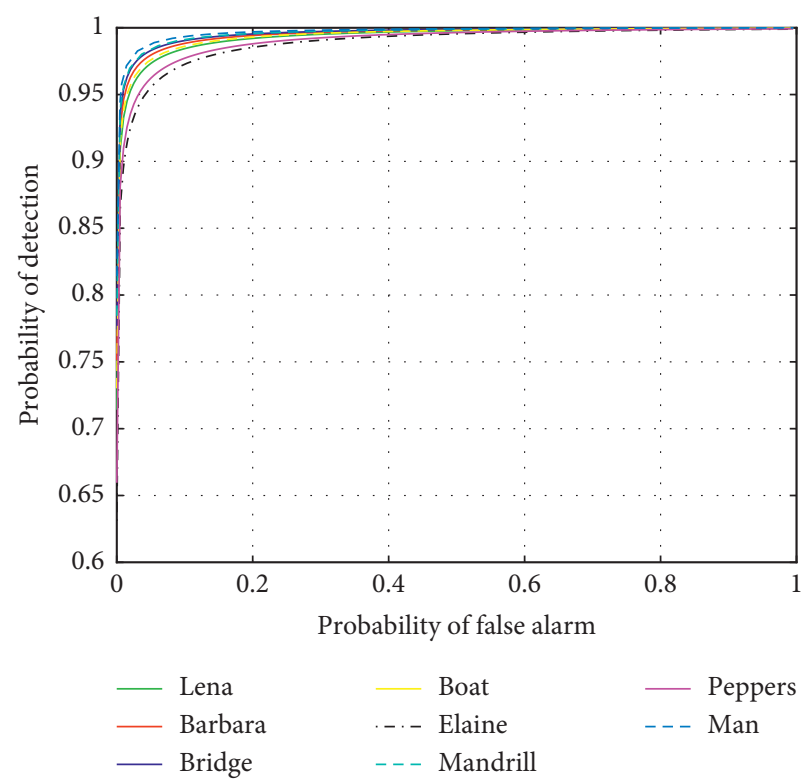

FIgURE 9: Performance of watermark detection for different images.

transformation and histogram equalization attack. These problems will be addressed by developing some matrix decomposition-based watermarking methods or deep learning-based watermarking algorithms in our future work.

\section{Conclusion}

We have developed an image watermarking algorithm by using the visual saliency model in the contourlet domain. In watermark embedding, high-energy image blocks are selected for the watermark embedding space, and the watermark embedded strength factor is exploited by taking advantage of texture masking and visual salience. The watermark can be embedded into the contourlet coefficients adaptively by using this strategy. For watermark decoding, the GGD model is used to describe the contourlet coefficients, and the ROC has been derived by applying the statistic signal processing method. Finally, we have performed several experiments to demonstrate the proposed method. Simulation results show that our watermarking 
method has satisfied imperceptibility and robustness. In the future work, a novel watermark detection approach will be designed using the deep learning or generative adversarial network method.

\section{Data Availability}

Eight standard grayscale images Lena, Barbara, Bridge, Boat, Elaine, Mandrill, Peppers, and Man are used as host images in the simulations, which are shown in Figure 4 in this paper. The results in this paper are entirely theoretical and analytical. The main steps of the demonstrations for each result are clearly reported in the text and the paper is fully consistent without the support of any additional data.

\section{Conflicts of Interest}

The authors declare that they have no conflicts of interest.

\section{Acknowledgments}

This work was supported by the Natural Science Foundation of Jiangxi (no. 20192BAB207013). The authors would like to thank professor M.N. Do for providing the CODE to perform the contourlet transform.

\section{References}

[1] M. Asikuzzaman and M. R. Pickering, "An overview of digital video watermarking," IEEE Transactions on Circuits and Systems for Video Technology, vol. 28, no. 9, pp. 2131-2153, 2018.

[2] H. Fang, W. Zhang, H. Zhou, H. Cui, and N. Yu, "Screenshooting resilient watermarking," IEEE Transactions on Information Forensics and Security, vol. 14, no. 6, pp. 1403-1418, 2019.

[3] S. Li and X. Zhang, "Toward construction-based data hiding: from secrets to fingerprint images," IEEE Transactions on Image Processing, vol. 28, no. 3, pp. 1482-1497, 2019.

[4] Y. Huang, B. Niu, H. Guan, and S. Zhang, "Enhancing image watermarking with adaptive embedding parameter and PSNR guarantee," IEEE Transactions on Multimedia, vol. 21, no. 10, pp. 2447-2460, 2019.

[5] D. Rajani and P. R. Kumar, "An optimized blind watermarking scheme based on principal component analysis in redundant discrete wavelet domain," Signal Processing, vol. 172, Article ID 107556, 2020.

[6] B. Chen, Y. Wu, G. Coatrieux, X. Chen, and Y. Zheng, "JSNet: a simulation network of JPEG lossy compression and restoration for robust image watermarking against JPEG attack," Computer Vision and Image Understanding, vol. 197-198, Article ID 103015, 2020.

[7] B. Xiao, J. Luo, X. Bi, W. Li, and B. Chen, "Fractional discrete Tchebyshev moments and their applications in image encryption and watermarking," Information Sciences, vol. 516, pp. 545-559, 2020.

[8] I. J. Cox, J. Kilian, F. T. Leighton, and T. Shamoon, "Secure spread spectrum watermarking for multimedia," IEEE Transactions on Image Processing, vol. 6, no. 12, pp. 16731687, 1997.

[9] Q. Cheng and T. S. Huang, "An additive approach to transform-domain information hiding and optimum detection structure," IEEE Transactions on Multimedia, vol. 3, no. 3, pp. 273-284, 2001.

[10] W. Liu, L. Dong, and W. Zeng, "Optimum detection for spread-spectrum watermarking that employs self-masking," IEEE Transactions on Information Forensics and Security, vol. 2, no. 4, pp. 645-654, 2007.

[11] R. Kwitt, P. Meerwald, and A. Uhl, "Lightweight detection of additive watermarking in the DWT-domain," IEEE Transactions on Image Processing, vol. 20, no. 2, pp. 474-484, 2011.

[12] L. Zhang and D. Wei, "Image watermarking based on matrix decomposition and gyrator transform in invariant integer wavelet domain," Signal Processing, vol. 169, Article ID 107421, 2020.

[13] B. Chen and G. W. Wornell, "Quantization index modulation: a class of provably good methods for digital watermarking and information embedding," IEEE Transactions on Information Theory, vol. 47, no. 4, pp. 1423-1443, 2001.

[14] F. Perez-Gonzalez, C. Mosquera, M. Barni, and A. Abrardo, "Rational dither modulation: a high-rate data-hiding method invariant to gain attacks," IEEE Transactions on Signal Processing, vol. 53, no. 10, pp. 3960-3975, 2005.

[15] Q. Li and I. J. Cox, "Using perceptual models to improve fidelity and provide resistance to valumetric scaling for quantization index modulation watermarking," IEEE Transactions on Information Forensics and Security, vol. 2, no. 2, pp. 127-139, 2007.

[16] N. K. Kalantari and S. M. Ahadi, "A logarithmic quantization index modulation for perceptually better data hiding," IEEE Transactions on Image Processing, vol. 19, no. 6, pp. 15041517, 2010.

[17] M. A. Akhaee, S. M. E. Sahraeian, and C. Jin, "Blind image watermarking using a sample projection approach," IEEE Transactions on Information Forensics and Security, vol. 6, no. 3, pp. 883-893, 2011.

[18] M. Zareian and H. R. Tohidypour, "A novel gain invariant quantization-based watermarking approach," IEEE Transactions on Information Forensics and Security, vol. 9, no. 11, pp. 1804-1813, 2014.

[19] N. Cai, N. Zhu, S. Weng, and B. Wing-Kuen Ling, "Difference angle quantization index modulation scheme for image watermarking," Signal Processing: Image Communication, vol. 34, pp. 52-60, 2015.

[20] J. Liu, Y. Xu, S. Wang, and C. Zhu, "Complex wavelet-domain image watermarking algorithm using \$\$L_1\$ L 1 -norm function-based quantization," Circuits, Systems, and Signal Processing, vol. 37, no. 3, pp. 1268-1286, 2018.

[21] M. Sadeghi, R. Toosi, and M. A. Akhaee, "Blind gain invariant image watermarking using random projection approach," Signal Processing, vol. 163, pp. 213-224, 2019.

[22] H. Fang, H. Zhou, Z. Ma, W. Zhang, and N. Yu, "A robust image watermarking scheme in DCT domain based on adaptive texture direction quantization," Multimedia Tools and Applications, vol. 78, no. 7, pp. 8075-8089, 2019.

[23] J. Wu, L. Li, W. Dong, G. Shi, W. Lin, and C.-C. J. Kuo, "Enhanced just noticeable difference model for images with pattern complexity," IEEE Transactions on Image Processing, vol. 26, no. 6, pp. 2682-2693, 2017.

[24] W. Wan, J. Wang, J. Li et al., "Pattern complexity-based JND estimation for quantization watermarking," Pattern Recognition Letters, vol. 130, pp. 157-164, 2020.

[25] H.-T. Hu, L.-Y. Hsu, and H.-H. Chou, "An improved SVDbased blind color image watermarking algorithm with mixed modulation incorporated," Information Sciences, vol. 519, pp. 161-182, 2020. 
[26] M. A. Akhaee, S. M. E. Sahraeian, B. Sankur, and F. Marvasti, "Robust scaling-based image watermarking using maximumlikelihood decoder with optimum strength factor," IEEE Transactions on Multimedia, vol. 11, no. 5, pp. 822-833, 2009.

[27] M. A. Akhaee, S. M. E. Sahraeian, and F. Marvasti, "Contourlet-based image watermarking using optimum detector in a noisy environment," IEEE Transactions on Image Processing, vol. 19, no. 4, pp. 967-980, 2010.

[28] H. Khalilian and I. V. Bajic, "Video watermarking with empirical PCA-based decoding," IEEE Transactions on Image Processing, vol. 22, no. 12, pp. 4825-4840, 2013.

[29] D. Bhowmik, M. Oakes, and C. Abhayaratne, "Visual attention-based image watermarking," IEEE Access, vol. 4, pp. 8002-8018, 2016.

[30] A. Cedillo-Hernandez, M. Cedillo-Hernandez, M. Nakano Miyatake, and H. Perez Meana, "A spatiotemporal saliencymodulated JND profile applied to video watermarking," Journal of Visual Communication and Image Representation, vol. 52, pp. 106-117, 2018.

[31] N. Yadav and K. Singh, "Robust image-adaptive watermarking using an adjustable dynamic strength factor," Signal, Image and Video Processing, vol. 9, no. 7, pp. 1531-1542, 2015.

[32] M. N. Do and M. Vetterli, "The contourlet transform: an efficient directional multiresolution image representation," IEEE Transactions on Image Processing, vol. 14, no. 12, pp. 2091-2106, 2005.

[33] X. Yang, W. Lin, Z. Liu, E. Ongg, and S. Yao, "Motioncompensated residue preprocessing in video coding based on just-noticeable-distortion profile," IEEE Transactions on Circuits and Systems for Video Technology, vol. 15, no. 6, pp. 745-752, 2005.

[34] L. Itti, C. Koch, and E. Niebur, "A model of saliency-based visual attention for rapid scene analysis," IEEE Transactions on Pattern Analysis and Machine Intelligence, vol. 20, no. 11, pp. 1254-1259, 1998.

[35] L. Itti, "Automatic foveation for video compression using a neurobiological model of visual attention," IEEE Transactions on Image Processing, vol. 13, no. 10, pp. 1304-1318, 2004.

[36] J. Wang, G. Liu, Y. Dai, J. Sun, Z. Wang, and S. Lian, "Locally optimum detection for barni's multiplicative watermarking in DWT domain," Signal Processing, vol. 88, no. 1, pp. 117-130, 2008.

[37] Z. Wang, A. C. Bovik, H. R. Sheikh, and E. P. Simoncelli, "Image quality assessment: from error visibility to structural similarity," IEEE Transactions on Image Processing, vol. 13, no. 4, pp. 600-612, 2004.

[38] F. Ernawan and M. N. Kabir, "A robust image watermarking technique with an optimal dct-psychovisual threshold," IEEE Access, vol. 6, pp. 20464-20480, 2018. 